\title{
Limits on Diffuse Dark Matter with HAWC
}

\section{Mora Durocher ${ }^{a, *}$ on behalf of the HAWC Collaboration}

(a complete list of authors can be found at the end of the proceedings)

\author{
${ }^{a}$ Physics Division, Los Alamos National Laboratory \\ Los Alamos, NM, USA \\ E-mail: mdurocher@lanl.gov
}

In addition to dense regions of dark matter, such as galaxy clusters and dwarf galaxies, dark matter annihilation and decay are also expected to have a nearly isotropic distribution across the sky. This isotropic component is less model-dependent than the flux from isolated dark matter targets, and would produce galactic contributions to the Diffuse Gamma-Ray Background (DGRB). With its continuous monitoring of the gamma-ray sky from a few hundred $\mathrm{GeV}$ to several hundred $\mathrm{TeV}$ and its wide field-of-view, the High Altitude Water Cherenkov (HAWC) observatory is well-suited to search for dark matter contributions in the DGRB. In this work, 535 days of HAWC data and Monte Carlo simulations were studied to set limits on annihilating or decaying diffuse dark matter at $\mathrm{TeV}$ energies. With this data, we consider both leptonic and hadronic dark matter channels and are able to constrain dark matter up to masses $>100 \mathrm{TeV}$.

$37^{\text {th }}$ International Cosmic Ray Conference (ICRC 2021)

July 12th - 23rd, 2021

Online - Berlin, Germany

\footnotetext{
*Presenter
} 


\section{Motivation and Detector}

Isotropic emission of gamma rays uncorrelated with any known sources are expected to be the main provenance of the Diffuse Gamma-Ray Background (DGRB). However, if dark matter were to annihilate or decay to produce gamma rays, due to the Earth's location near the middle of the galactic dark matter halo, a diffuse gamma-ray emission would be observed in all directions. This is particularly true if the DGRB is observed at multi-TeV energies where extra-galactic emission is attenuated via pair-production on the extra-galactic background light, making dark matter a more likely candidate for any observed high-energy DGRB emission.

In this work we use 535 days of data from the High Altitude Water Cherenkov (HAWC) gammaray observatory, taken from November 2014 to June 2016. With its 300 water Cherenkov detectors, HAWC was built to detect gamma rays in the energy range between $300 \mathrm{GeV}$ to more than 100 TeV. For ground-based detectors, such as HAWC, hadronic Cosmic Rays (CRs) are the main source of background to high-energy photon observation. Fortunately, above several TeV, the air showers produced by high-energy CRs and gamma rays differ in shape. By quantifying the smoothness of the lateral charge distribution function of air showers [4] most of the cosmic ray contamination can be removed.

\section{Analysis}

We implement a 2D binning scheme [1] focusing on high energy events with reconstructed energies above $10 \mathrm{TeV}$ and where more than $61.8 \%$ of the PMTs available were hit. Although this analysis does not include more recent HAWC data, the characteristics of the dataset used are very well known. A parallel work in this conference ${ }^{1}$ relies on the same data and contains more details on the methodology.

Previous studies have been performed using the HAWC observatory to set limits on the DGRB [5-7]. In this work, the DGRB region of interest is a strip centered on the Crab nebula's location. Within the strip, bright known $\gamma$-ray sources - i.e. the Crab, Geminga and the Galactic Plane have been removed to avoid contamination. The resulting DGRB strip has an area of $0.57 \mathrm{sr}$ and is shown in Figure 1.

As we apply tighter gamma/hadron separation cuts, few events remain and we must rely on Poisson statistics. For a binned likelihood analysis, the log-likelihood is calculated as the sum of the log of the Poisson probability to observe $N^{o b s}$ events in a bin given that the model predicts $N^{\text {pred }}$

$$
\ln \mathcal{L}=\sum_{i}^{\text {bins }} N_{i}^{\text {obs }} \log \left(N_{i}^{\text {pred }}\right)-\sum_{i}^{\text {bins }} N_{i}^{\text {pred }}
$$

where $N^{o b s}$ is set as the number of events in our DGRB strip and $N^{\text {pred }}$ depends on the number of Monte Carlo simulated events. We inject the latter with isotropic and spatial-model independent gamma-ray emissions from galactic dark matter interactions into tau leptons and bottom quarks [8]. However we do not perform a joint likelihood; in order to calculate the best estimate for the overall scale of the spectrum, the bins are not summed but treated as separate independent "experiments".

${ }^{1}$ https://pos.sissa.it/395/829 


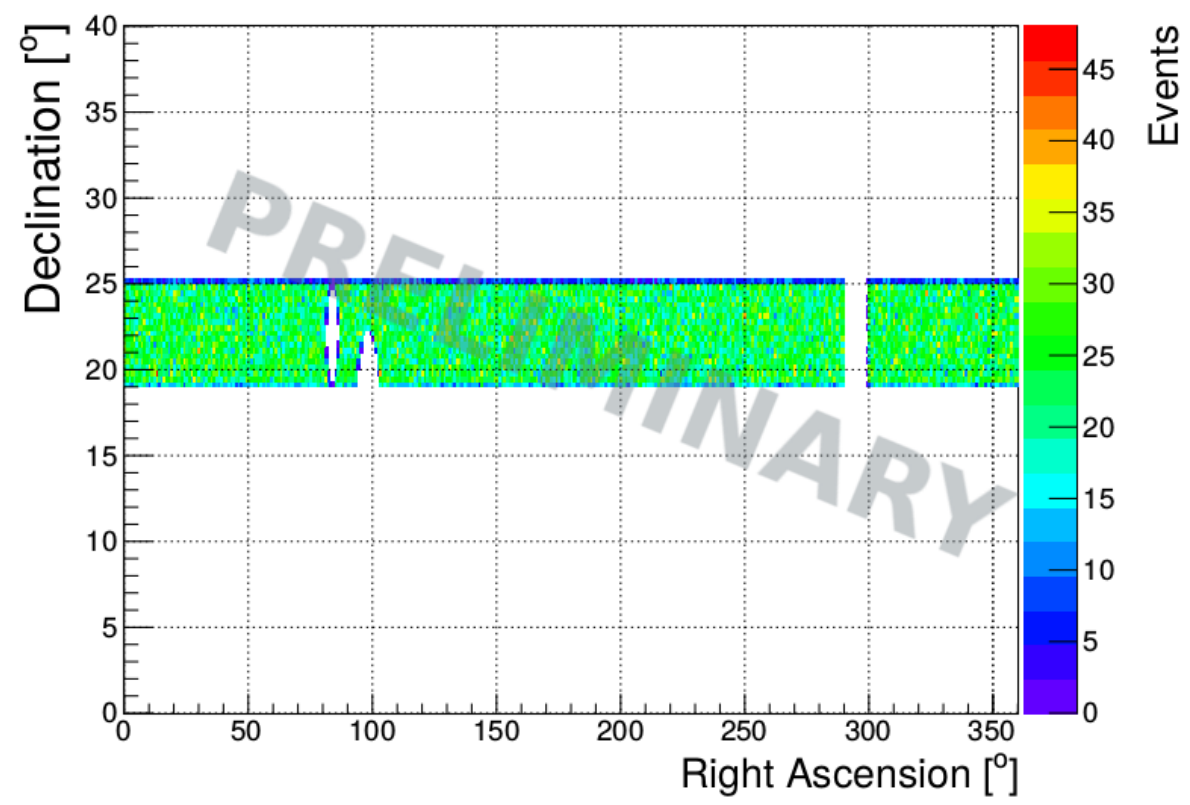

Figure 1: Map of the Diffuse Gamma-Ray Background strip

The $95 \%$ containment level of the maximum likelihood best estimate is calculated in each bin and the one with the lowest value is selected, as it would be the one with the most expansive limit.

\section{Results}

There are a few candidates which can be evaluated when it comes to dark matter masses and dark matter annihilation or decay spectra. We explore the more conventional channels for dark matter annihilation and decay interactions, which are the $b \bar{b}$ and $\tau^{+} \tau^{-}$channels. Both of these processes would lead to the creation of gamma rays. By injecting the spectra of chosen dark matter masses in our Monte Carlo simulation we can calculate the $95 \%$ containment level of the best estimate of the overall scale of said spectra, which is referred to as $\beta_{95 \%}$. We then chose the best $\beta_{95 \%}$ to calculate $\beta_{95 \%} \times\langle\sigma v\rangle$ for annihilation upper limits and the best $\beta_{95 \%}$ to calculate lifetime $\div \beta_{95 \%}$ for decay lower limits. The results are shown in Figure 2 .

\section{Discussion and Prospects}

It is possible that diffuse $\gamma$-ray emissions originate from annihilating or decaying dark matter in the extended halo around the Galaxy. Gamma rays would then be observed in all directions, as a background to all other $\gamma$-ray observations. The limits obtained here are pushing into an energy range higher than the Fermi-LAT Isotropic Gamma-Ray Background and the HAWC Andromeda Galaxy constraints, nonetheless we can observe better limits for interactions involving tau leptons when compared to the HAWC Burkert Galactic Halo limits.

With the addition of more years of data, upcoming improvements to the HAWC reconstruction algorithms and analytical methods, and the deployment of the outrigger array in 2018, stronger 

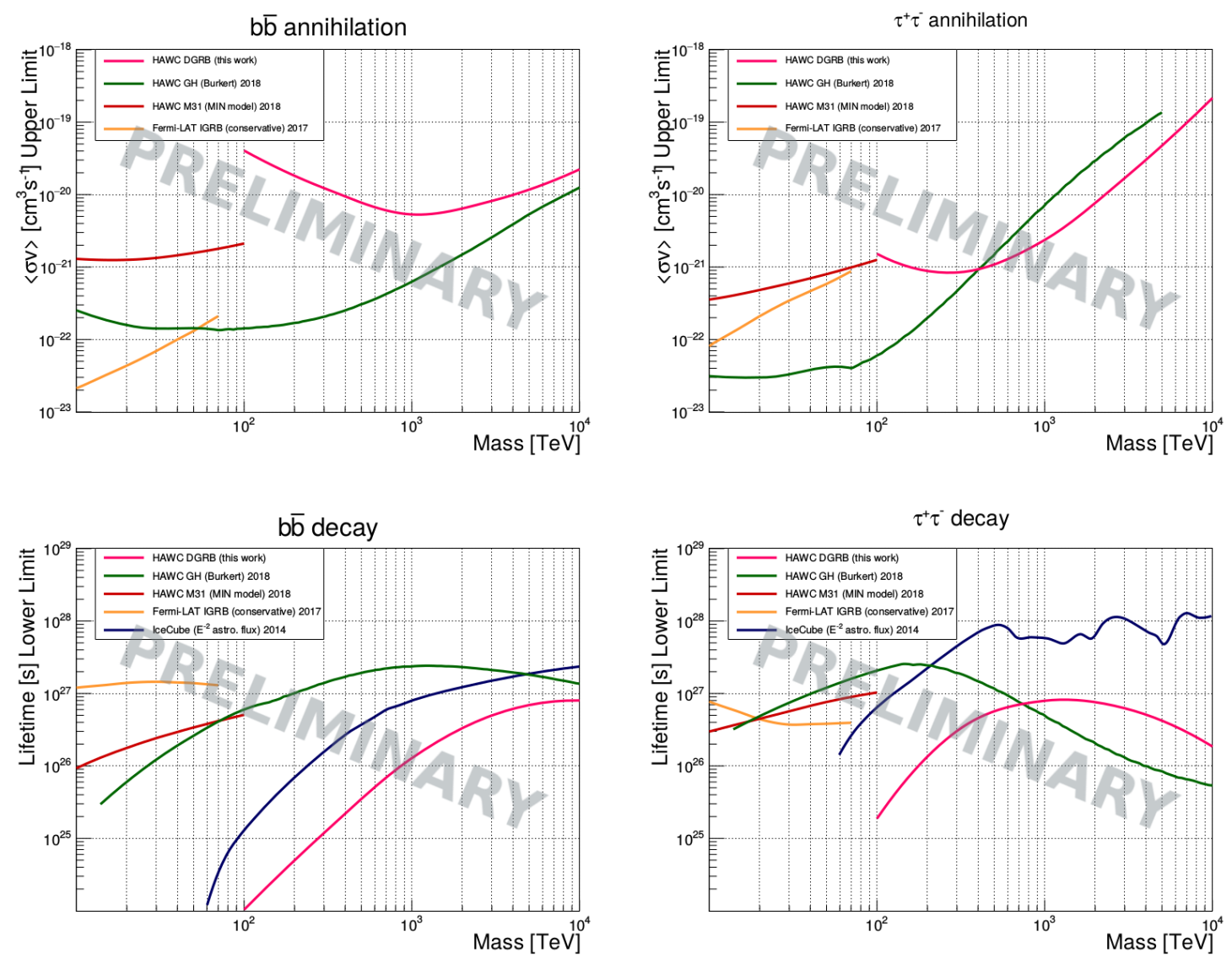

Figure 2: $95 \%$ confidence level limits on diffuse dark matter compared to recent dark matter searches with HAWC [9][10] and other experiments such as IceCube [11] and Fermi-LAT [12].

constraints on the previous results are expected. Furthermore, a next-generation Southern Widefield Gamma-ray Observatory (SWGO) ${ }^{2}$ is being considered for the Southern Hemisphere which will extend sensitivity to energies above the tens of PeV.

\section{Acknowledgments}

We acknowledge the support from: the US National Science Foundation (NSF); the US Department of Energy Office of High-Energy Physics; the Laboratory Directed Research and Development (LDRD) program of Los Alamos National Laboratory; Consejo Nacional de Ciencia y Tecnología (CONACyT), México, grants 271051, 232656, 260378, 179588, 254964, 258865, 243290, 132197, A1-S-46288, A1-S-22784, cátedras 873, 1563, 341, 323, Red HAWC, México; DGAPA-UNAM grants IG101320, IN111716-3, IN111419, IA102019, IN110621, IN110521; VIEP-BUAP; PIFI 2012, 2013, PROFOCIE 2014, 2015; the University of Wisconsin Alumni Research Foundation; the Institute of Geophysics, Planetary Physics, and Signatures at Los Alamos National Laboratory; Polish Science Centre grant, DEC-2017/27/B/ST9/02272; Coordinación de la Investigación Científica

${ }^{2}$ Www.swgo.org 
de la Universidad Michoacana; Royal Society - Newton Advanced Fellowship 180385; Generalitat Valenciana, grant CIDEGENT/2018/034; Chulalongkorn University's CUniverse (CUAASC) grant; Coordinación General Académica e Innovación (CGAI-UdeG), PRODEP-SEP UDG-CA499; Institute of Cosmic Ray Research (ICRR), University of Tokyo, H.F. acknowledges support by NASA under award number 80GSFC21M0002. We also acknowledge the significant contributions over many years of Stefan Westerhoff, Gaurang Yodh and Arnulfo Zepeda Dominguez, all deceased members of the HAWC collaboration. Thanks to Scott Delay, Luciano Díaz and Eduardo Murrieta for technical support.

\section{References}

[1] A.U. Abeysekara, A. Albert, R. Alfaro, C. Alvarez, J.D. Álvarez, J.R.A. Camacho et al., Measurement of the Crab Nebula Spectrum Past $100 \mathrm{TeV}$ with HAWC, The Astrophysical Journal 881 (2019) 134.

[2] A. Abeysekara et al., On the sensitivity of the HAWC observatory to gamma-ray bursts, Astroparticle Physics 35 (2012) 641 .

[3] A. Abeysekara et al., Sensitivity of the High Altitude Water Cherenkov Detector to Sources of Multi-TeV Gamma Rays, Astropart. Phys. 50-52 (2013) 26 [1306. 5800].

[4] A. Abeysekara et al., Observation of the Crab Nebula with the HAWC Gamma-Ray Observatory, Astrophys. J. 843 (2017) 39 [1701.01778].

[5] J. Pretz, Limit on an Isotropic Diffuse Gamma-Ray Population with HAWC, 2015.

[6] H. Zhou, C.D. Rho and G. Vianello, Probing Galactic Diffuse TeV Gamma-Ray Emission with the HAWC Observatory, 2017.

[7] J.P. Harding, Constraints on the Diffuse Gamma-Ray Background with HAWC, 2019.

[8] C.W. Bauer, N.L. Rodd and B.R. Webber, Dark Matter Spectra from the Electroweak to the Planck Scale, 2020.

[9] A. Abeysekara, A. Albert, R. Alfaro, C. Alvarez, R. Arceo, J. Arteaga-Velázquez et al., A search for dark matter in the Galactic halo with HAWC, Journal of Cosmology and Astroparticle Physics 2018 (2018) 049-049.

[10] A. Albert, R. Alfaro, C. Alvarez, J. Álvarez, R. Arceo, J. Arteaga-Velázquez et al., Search for dark matter gamma-ray emission from the Andromeda Galaxy with the High-Altitude Water Cherenkov Observatory, Journal of Cosmology and Astroparticle Physics 2018 (2018) 043-043.

[11] A. Esmaili, S.K. Kang and P.D. Serpico, IceCube events and decaying dark matter: hints and constraints, Journal of Cosmology and Astroparticle Physics 2014 (2014) 054-054.

[12] W. Liu, X.-J. Bi, S.-J. Lin and P.-F. Yin, Constraints on dark matter annihilation and decay from the isotropic gamma-ray background, Chinese Physics C 41 (2017) 045104. 


\section{Full Authors List: HAWC Collaboration}

A.U. Abeysekara ${ }^{48}$, A. Albert ${ }^{21}$, R. Alfaro ${ }^{14}$, C. Alvarez ${ }^{41}$, J.D. Álvarez ${ }^{40}$, J.R. Angeles Camacho ${ }^{14}$, J.C. Arteaga-Velázquez ${ }^{40}$, K. P. Arunbabu $^{17}$, D. Avila Rojas ${ }^{14}$, H.A. Ayala Solares ${ }^{28}$, R. Babu ${ }^{25}$, V. Baghmanyan ${ }^{15}$, A.S. Barber ${ }^{48}$, J. Becerra Gonzalez ${ }^{11}$, E. BelmontMoreno $^{14}$, S.Y. BenZvi ${ }^{29}$, D. Berley ${ }^{39}$, C. Brisbois ${ }^{39}$, K.S. Caballero-Mora ${ }^{41}$, T. Capistrán ${ }^{12}$, A. Carramiñana ${ }^{18}$, S. Casanova ${ }^{15}$, O. Chaparro-Amaro $^{3}$, U. Cotti ${ }^{40}$, J. Cotzomi $^{8}$, S. Coutiño de León ${ }^{18}$, E. De la Fuente ${ }^{46}$, C. de León ${ }^{40}$, L. Diaz-Cruz ${ }^{8}$, R. Diaz Hernandez ${ }^{18}$, J.C. Díaz-Vélez ${ }^{46}$, B.L. Dingus ${ }^{21}$, M. Durocher ${ }^{21}$, M.A. DuVernois ${ }^{45}$, R.W. Ellsworth ${ }^{39}$, K. Engel ${ }^{39}$, C. Espinoza ${ }^{14}$, K.L. Fan ${ }^{39}$, K. Fang $^{45}$, M. Fernández Alonso ${ }^{28}$, B. Fick ${ }^{25}$, H. Fleischhack ${ }^{51,11,52}$, J.L. Flores ${ }^{46}$, N.I. Fraija ${ }^{12}$, D. Garcia ${ }^{4}$, J.A. García-González ${ }^{20}$, J. L. García-Luna ${ }^{46}$, G. García-Torales ${ }^{46}$, F. Garfias ${ }^{12}$, G. Giacinti ${ }^{22}$, H. Goksu ${ }^{22}$, M.M. González ${ }^{12}$, J.A. Goodman ${ }^{39}$, J.P. Harding ${ }^{21}$, S. Hernandez $^{14}$, I. Herzog ${ }^{25}$, J. Hinton ${ }^{22}$, B. Hona ${ }^{48}$, D. Huang ${ }^{25}$, F. Hueyotl-Zahuantitla ${ }^{41}$, C.M. Hui ${ }^{23}$, B. Humensky ${ }^{39}$, P. Hüntemeyer ${ }^{25}$, A. Iriarte ${ }^{12}$, A. Jardin-Blicq ${ }^{22,49,50}$, H. Jhee ${ }^{43}$, V. Joshi ${ }^{7}$, D. Kieda ${ }^{48}$, G J. Kunde ${ }^{21}$, S. Kunwar ${ }^{22}$, A. Lara ${ }^{17}$, J. Lee ${ }^{43}$, W.H. Lee ${ }^{12}$, D. Lennarz ${ }^{9}$, H. León Vargas ${ }^{14}$, J. Linnemann ${ }^{24}$, A.L. Longinotti ${ }^{12}$, R. López-Coto ${ }^{19}$, G. Luis-Raya ${ }^{44}$, J. Lundeen ${ }^{24}$, K. Malone ${ }^{21}$, V. Marandon $^{22}$, O. Martinez ${ }^{8}$, I. Martinez-Castellanos ${ }^{39}$, H. Martínez-Huerta ${ }^{38}$, J. Martínez-Castro ${ }^{3}$, J.A.J. Matthews ${ }^{42}$, J. McEnery ${ }^{11}$, P. Miranda-Romagnoli $^{34}$, J.A. Morales-Soto ${ }^{40}$, E. Moreno ${ }^{8}$, M. Mostafá ${ }^{28}$, A. Nayerhoda ${ }^{15}$, L. Nellen ${ }^{13}$, M. Newbold ${ }^{48}$, M.U. Nisa ${ }^{24}$, R. Noriega-Papaqui ${ }^{34}$, L. Olivera-Nieto ${ }^{22}$, N. Omodei ${ }^{32}$, A. Peisker ${ }^{24}$, Y. Pérez Araujo ${ }^{12}$, E.G. Pérez-Pérez ${ }^{44}$, C.D. Rho ${ }^{43}$, C. Rivière ${ }^{39}$, D. Rosa-Gonzalez $^{18}$, E. Ruiz-Velasco ${ }^{22}$, J. Ryan ${ }^{26}$, H. Salazar ${ }^{8}$, F. Salesa Greus ${ }^{15,53}$, A. Sandoval ${ }^{14}$, M. Schneider ${ }^{39}$, H. Schoorlemmer ${ }^{22}$, J. Serna-Franco ${ }^{14}$, G. Sinnis ${ }^{21}$, A.J. Smith ${ }^{39}$, R.W. Springer ${ }^{48}$, P. Surajbali ${ }^{22}$, I. Taboada ${ }^{9}$, M. Tanner ${ }^{28}$, K. Tollefson ${ }^{24}$, I. Torres ${ }^{18}$, R. Torres-Escobedo $^{30}$, R. Turner ${ }^{25}$, F. Ureña-Mena ${ }^{18}$, L. Villaseñor ${ }^{8}$, X. Wang ${ }^{25}$, I.J. Watson ${ }^{43}$, T. Weisgarber ${ }^{45}$, F. Werner ${ }^{22}$, E. Willox ${ }^{39}$, J. Wood ${ }^{23}$, G.B. Yodh ${ }^{35}$, A. Zepeda ${ }^{4}$, H. Zhou ${ }^{30}$

${ }^{1}$ Barnard College, New York, NY, USA, ${ }^{2}$ Department of Chemistry and Physics, California University of Pennsylvania, California, PA, USA, ${ }^{3}$ Centro de Investigación en Computación, Instituto Politécnico Nacional, Ciudad de México, México, ${ }^{4}$ Physics Department, Centro de Investigación y de Estudios Avanzados del IPN, Ciudad de México, México, ${ }^{5}$ Colorado State University, Physics Dept., Fort Collins, CO, USA, ${ }^{6}$ DCI-UDG, Leon, Gto, México, ${ }^{7}$ Erlangen Centre for Astroparticle Physics, Friedrich Alexander Universität, Erlangen, BY, Germany, ${ }^{8}$ Facultad de Ciencias Físico Matemáticas, Benemérita Universidad Autónoma de Puebla, Puebla, México, ${ }^{9}$ School of Physics and Center for Relativistic Astrophysics, Georgia Institute of Technology, Atlanta, GA, USA, ${ }^{10}$ School of Physics Astronomy and Computational Sciences, George Mason University, Fairfax, VA, USA, ${ }^{11}$ NASA Goddard Space Flight Center, Greenbelt, MD, USA, ${ }^{12}$ Instituto de Astronomía, Universidad Nacional Autónoma de México, Ciudad de México, México, ${ }^{13}$ Instituto de Ciencias Nucleares, Universidad Nacional Autónoma de México, Ciudad de México, México, ${ }^{14}$ Instituto de Física, Universidad Nacional Autónoma de México, Ciudad de México, México, ${ }^{15}$ Institute of Nuclear Physics, Polish Academy of Sciences, Krakow, Poland, ${ }^{16}$ Instituto de Física de São Carlos, Universidade de São Paulo, São Carlos, SP, Brasil, ${ }^{17}$ Instituto de Geofísica, Universidad Nacional Autónoma de México, Ciudad de México, México, ${ }^{18}$ Instituto Nacional de Astrofísica, Óptica y Electrónica, Tonantzintla, Puebla, México, ${ }^{19}$ INFN Padova, Padova, Italy, ${ }^{20}$ Tecnologico de Monterrey, Escuela de Ingeniería y Ciencias, Ave. Eugenio Garza Sada 2501, Monterrey, N.L., 64849, México, ${ }^{21}$ Physics Division, Los Alamos National Laboratory, Los Alamos, NM, USA, ${ }^{22}$ Max-Planck Institute for Nuclear Physics, Heidelberg, Germany, ${ }^{23}$ NASA Marshall Space Flight Center, Astrophysics Office, Huntsville, AL, USA, ${ }^{24}$ Department of Physics and Astronomy, Michigan State University, East Lansing, MI, USA, ${ }^{25}$ Department of Physics, Michigan Technological University, Houghton, MI, USA, ${ }^{26}$ Space Science Center, University of New Hampshire, Durham, NH, USA, ${ }^{27}$ The Ohio State University at Lima, Lima, OH, USA, ${ }^{28}$ Department of Physics, Pennsylvania State University, University Park, PA, USA, ${ }^{29}$ Department of Physics and Astronomy, University of Rochester, Rochester, NY, USA, ${ }^{30}$ Tsung-Dao Lee Institute and School of Physics and Astronomy, Shanghai Jiao Tong University, Shanghai, China, ${ }^{31}$ Sungkyunkwan University, Gyeonggi, Rep. of Korea, ${ }^{32}$ Stanford University, Stanford, CA, USA, ${ }^{33}$ Department of Physics and Astronomy, University of Alabama, Tuscaloosa, AL, USA, ${ }^{34}$ Universidad Autónoma del Estado de Hidalgo, Pachuca, Hgo., México, ${ }^{35}$ Department of Physics and Astronomy, University of California, Irvine, Irvine, CA, USA, ${ }^{36}$ Santa Cruz Institute for Particle Physics, University of California, Santa Cruz, Santa Cruz, CA, USA, ${ }^{37}$ Universidad de Costa Rica, San José, Costa Rica, ${ }^{38}$ Department of Physics and Mathematics, Universidad de Monterrey, San Pedro Garza García, N.L., México, ${ }^{39}$ Department of Physics, University of Maryland, College Park, MD, USA, ${ }^{40}$ Instituto de Física y Matemáticas, Universidad Michoacana de San Nicolás de Hidalgo, Morelia, Michoacán, México, ${ }^{41}$ FCFM-MCTP, Universidad Autónoma de Chiapas, Tuxtla Gutiérrez, Chiapas, México, ${ }^{42}$ Department of Physics and Astronomy, University of New Mexico, Albuquerque, NM, USA, ${ }^{43}$ University of Seoul, Seoul, Rep. of Korea, ${ }^{44}$ Universidad Politécnica de Pachuca, Pachuca, Hgo, México, ${ }^{45}$ Department of Physics, University of Wisconsin-Madison, Madison, WI, USA, ${ }^{46}$ CUCEI, CUCEA, Universidad de Guadalajara, Guadalajara, Jalisco, México, ${ }^{47}$ Universität Würzburg, Institute for Theoretical Physics and Astrophysics, Würzburg, Germany, ${ }^{48}$ Department of Physics and Astronomy, University of Utah, Salt Lake City, UT, USA, ${ }^{49}$ Department of Physics, Faculty of Science, Chulalongkorn University, Pathumwan, Bangkok 10330, Thailand, ${ }^{50}$ National Astronomical Research Institute of Thailand (Public Organization), Don Kaeo, MaeRim, Chiang Mai 50180, Thailand, ${ }^{51}$ Department of Physics, Catholic University of America, Washington, DC, USA, ${ }^{52}$ Center for Research and Exploration in Space Science and Technology, NASA/GSFC, Greenbelt, MD, USA, ${ }^{53}$ Instituto de Física Corpuscular, CSIC, Universitat de València, Paterna, Valencia, Spain 